\title{
DIVISION XII / COMMISSION 5 / WORKING GROUP FITS DATA FORMAT
}

\author{
CHAIR \\ VICE-CHAIR \\ PAST CHAIR \\ MEMBERS
}

\author{
William D. Pence \\ François Ochsenbein \\ Donald C. Wells \\ Steven W. Allen, Mark R. Calabretta, \\ Lucio Chiappetti, Daniel Durand, \\ Thierry Forveille, Carlos Gabriel, \\ Eric Greisen, Preben J. Grosbol, \\ Robert J. Hanisch, Walter J. Jaffe, \\ Osamu Kanamitsu, Oleg Yu. Malkov, \\ Clive G. Page, Arnold H. Rots, \\ Richard A. Shaw, Elizabeth Stobie, \\ William T. Thompson, Douglas C. Tody, \\ Andreas Wicenec
}

TRIENNIAL REPORT 2006 - 2009

\section{Introduction}

The Working Group FITS (WG-FITS) is the international control authority for the Flexible Image Transport System (FITS) data format. The WG-FITS was formed in 1988 by a formal resolution of the IAU XX General Assembly in Baltimore (MD, USA), 1988, to maintain the existing FITS standards and to approve future extensions to FITS.

\section{Developments within the past triennium}

The two most significant activities of the WG during the 2006 - 2009 triennium were to produce a new version of the formal FITS standard document, which precisely defines the requirements of the FITS format, and to create a new registry for documenting existing FITS conventions. These activities are described in the following two subsections.

\subsection{New FITS standard document}

The requirements of the FITS format are formally defined in the FITS standard document which is controlled by the WG-FITS. The last major revision to this document was made in 1999, so in early 2007, a small subcommittee (L. Chiappetti, C. Page, W. Pence, R. Shaw, and E. Stobie) was appointed to consider ways to update the document. After several months of work, this subcommittee produced a new draft of the FITS standard which, while not proposing any major changes to the FITS requirements, did contain dozens of small technical changes, as well as hundreds of other editorial changes to better organize and clarify many sections in the document. One significant change was the addition of a new section that summarizes the conventions for representing world coordinate information, such as in defining the position of an image on the celestial 
sphere. This information was previously only available in three separately published papers.

This proposed new FITS standard document went through a rigorous review process starting with a public comment period on the official FITS newsgroup, <sci.astro.fits>, from July through October 2007. A slightly revised version of the document was then formally endorsed by the four regional FITS committees (representing North America, Europe, Japan, and Australia \& New Zealand) in February 2008. This was then followed by a final review by the WG-FITS which formally approved this new version of the FITS standard in July 2008. It is now publicly available on the FITS Support Office web site at <fits.gsfc.nasa.gov>.

\subsection{Registry of FITS conventions}

The other main activity of the WG-FITS during this period was to create a registry on the FITS Support Office Web site for documenting the many existing FITS conventions that are in use within the FITS community. These conventions have been independently developed by many different groups. The registry provides a convenient central location for documenting the various FITS keywords or other FITS data structures that are used by each convention. The registration process is intentionally quite simple, so unlike changes to the FITS standard which can take years to complete, a convention can be entered into the registry in a matter of weeks. There is a required public comment period before the convention is registered, but this is mainly just to ensure that the documentation about the convention is sufficiently clear and complete.

As of July 2008, the following conventions have been registered:

- CONTINUE Long String Keyword convention for writing string keyword values that are longer than the 68-character limit of a single FITS keyword.

- FOREIGN file encapsulation for wrapping other types of files in FITS.

- CHECKSUM keyword convention for verifying the integrity of FITS HDUs.

- INHERIT keyword which indicates that a HDU should inherit the primary header keywords.

- Column Limits keywords (TLMINn/TLMAXn and TDMINn/TDMAXn) which define the legal and actual minimum and maximum values in a table column.

- Tiled Image Compression convention for dividing an image into a grid of tiles and then storing each compressed tile in a variable length array column of a binary table.

- ESO HIERARCH Keyword convention uses a hierarchical structure to define the keyword name. This convention can be generalized to support keyword names longer than 8 characters or containing characters that would not be allowed in a standard FITS keyword name.

- Euro3D interchange data format for integral field spectroscopy in which 1-dimensional spectra are obtained at multiple positions over a 2-dimensional spatial field of view.

- OIFITS data format for optical interferometry.

- Multi-Beam FITS (MBFITS) data format for single-dish mm/submm telescopes.

- Hierarchical Grouping convention for defining hierarchical associations of HDUs.

Several more conventions are pending, and it is expected that the registry will continue to grow each year.

\section{Future plans}

There are a number of other important FITS issues that need to be addressed by the WG-FITS in the next triennium. Some of these are 
- Develop standard conventions for specifying time and date coordinates.

- Complete the draft specification for representing distortions in world coordinate systems.

- Rewrite the FITS Users Guide which is currently obsolete.

- Consider elevating some of the conventions that are documented in the FITS registry to the status of a full FITS standard.

These activities are certain to keep the WG-FITS busy for the foreseeable future.

William D. Pence chair of the Working Group 